\title{
Rapid recognition and treatment of cerebral air embolism: The role of neuromonitoring
}

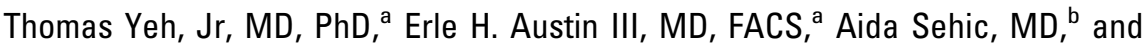

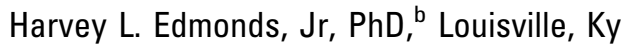

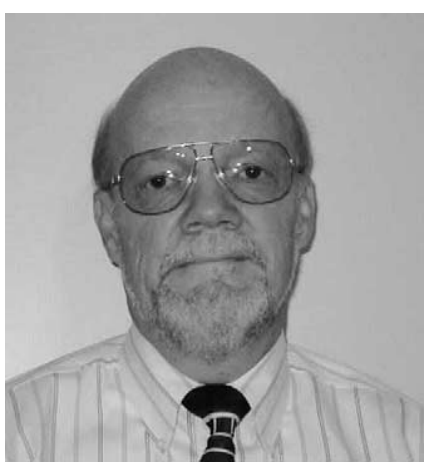

Dr Harvey L. Edmonds

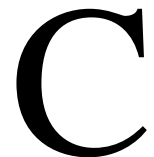

erebral air embolism is a rare but serious complication of cardiac operations. ${ }^{1}$ Transcranial Doppler ultrasonography is a sensitive monitor of gas bubble entry into the cerebral circulation. Here we describe a case of cerebral air embolism during a Fontan procedure and the role of transcranial Doppler ultrasonography, cerebral oximetry, and electroencephalography (EEG) in its recognition and management.

\section{Clinical Summary}

A 27-month-old boy with an unbalanced atrioventricular canal (hypoplastic left ventricle and dominant right ventricle) and hypoplastic aortic arch underwent the Norwood procedure at 5 days of age. The preoperative Fontan evaluation revealed trivial common atrioventricular valve regurgitation, good ventricular function, low pulmonary artery pressures, no pulmonary artery stenoses, and a left superior vena cava that was closed before the Fontan procedure.

Routine intraoperative monitoring included transesophageal echocardiography, femoral arterial and central venous pressure, electrocardiography, pulse oximetry, 8-channel EEG, right middle cerebral artery transcranial Doppler ultrasonography, and bihemispheric transcranial cerebral oximetry (spatially resolved, dualwavelength, near-infrared reflectance spectroscopy).

After aortic cannulation, cardiopulmonary bypass was initiated. With the heart beating, an extracardiac nonfenestrated Fontan connection was constructed with pedicled pericardium. ${ }^{2}$ After weaning from cardiopulmonary bypass, increased central venous pressure (22 $\mathrm{mm} \mathrm{Hg}$ with a left atrial pressure of $5 \mathrm{~mm} \mathrm{Hg}$ ) and low systemic blood pressure and oxygen saturation prompted resumption of cardiopulmonary bypass for Fontan fenestration.

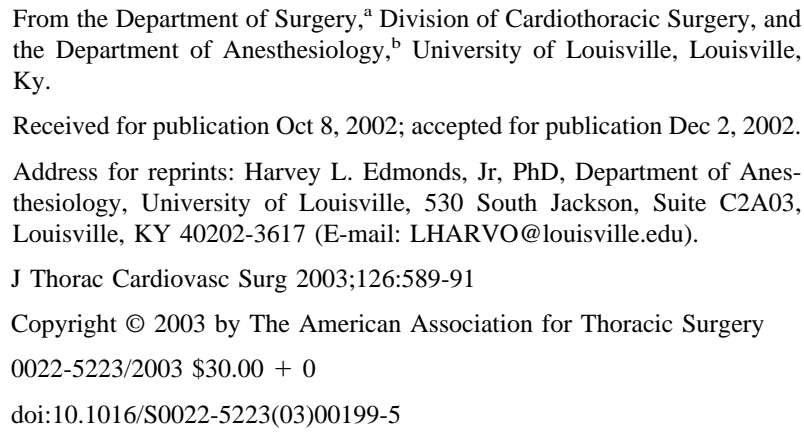

Under fibrillatory arrest, the extracardiac (pericardial) tunnel was opened, the free right atrial wall was opened, and a polytetrafluoroethylene patch (Gore-Tex; W. L. Gore \& Associates, Inc, Flagstaff, Ariz) with a 4-mm fenestration was inserted in the right atrial free wall.

Despite continued fibrillatory arrest, cerebral air embolism of uncertain cause was detected by means of transcranial Doppler ultrasonography (Figure 1, $A$ ). In addition, cerebral oxygen saturation declined precipitously (Figure 2, $A$ ); and artifacts appeared in the EEG (Figure 2, B).

Neuroprotective strategies were initiated, including deep Trendelenberg positioning, packing the head in ice, ascending aorta venting, rapid systemic cooling to EEG near-silence (Figure 2, $B$ ), and brief circulatory arrest (Figure $1, B$ ) before initiation of retrograde cerebral perfusion (Figure 1, $C$ ). Antegrade cerebral perfusion was restored after substantial gas bubble evacuation through the aortic vent, return of cerebral blood flow (Figure 1,D), and improved cerebral oxygen saturation (Figure 2, A). Spontaneous EEG activity returned quickly after rewarming (Figure 2, $B$ ). However, new asymmetries were evident in cerebral oxygen saturation (Figure 2, $A$ ) and EEG findings (Figure 2, B). The right hemisphere evidenced dysoxygenation and EEG suppression. Because of these asymmetries, a prophylactic neuroprotective dose of fosphenytoin $(10 \mathrm{mg} / \mathrm{kg})$ was administered. ${ }^{3}$ At sternal closure, the asymmetries had nearly disappeared. A neurologic examination the following day revealed no new focal neurologic or EEG abnormality (Figure 2, B).

\section{Comments}

Transcranial Doppler ultrasonography is exquisitely sensitive to the presence of air bubbles in the cerebral circulation. Because some emboliform ultrasonic high-intensity signals are often detected in operations requiring the opening of a cardiac chamber, additional neurophysiologic monitoring is essential to discriminate between benign and potentially pathologic embolization. Sudden rapid cerebral oxygen desaturation and coincident global loss of EEG activity facilitate this discrimination. In the present case, without multimodality neuromonitoring, the surgical team would have been completely unaware of the cerebral air embolism.

Two essential treatment elements for cerebral air embolism are neuroprotection and flow restoration. Although neuroprotection by means of hypothermia is well established, the effectiveness of gas bubble aspiration from the aorta after retrograde cerebral perfusion 

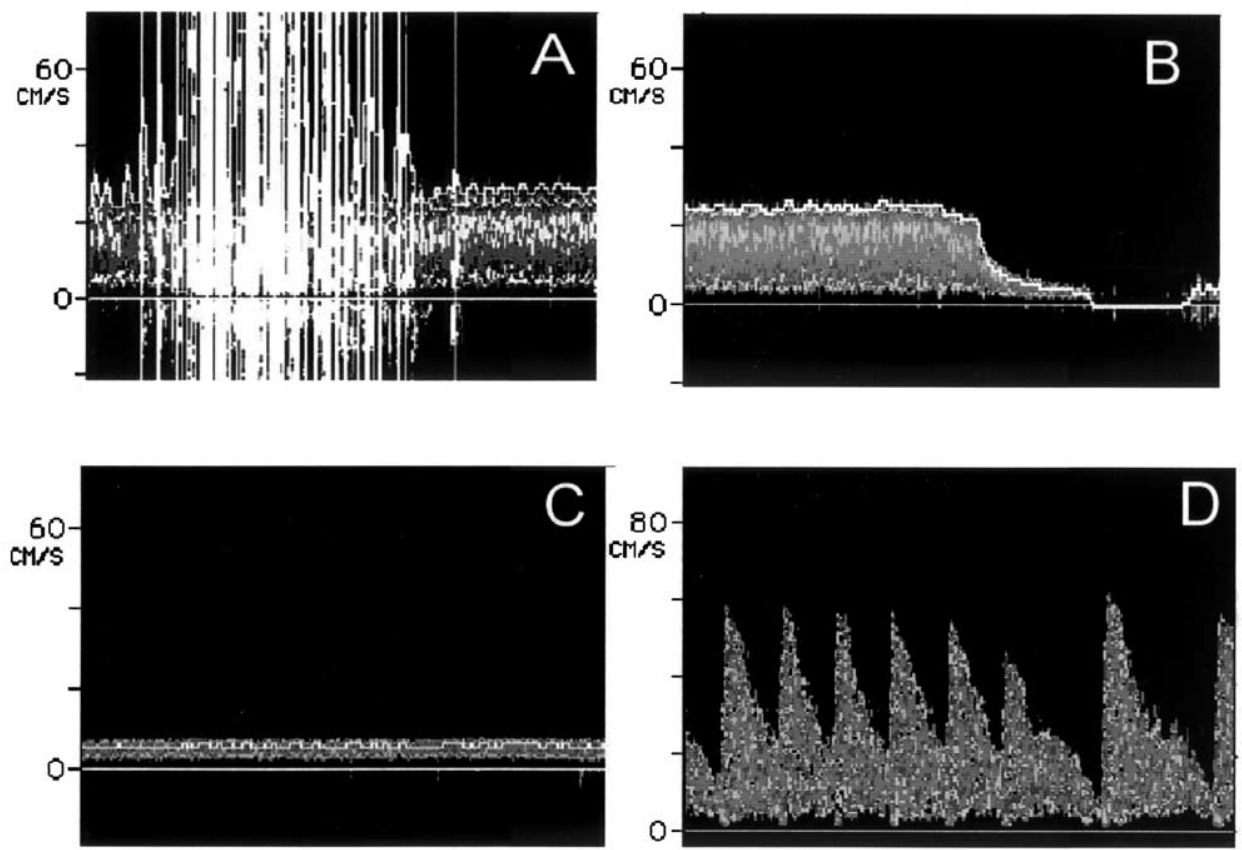

Figure 1. Noteworthy changes in the right middle cerebral artery flow-velocity spectrum associated with air embolism and its successful treatment. A, The sudden appearance of high-intensity transient signals (intense, white, full-scale vertical deflections) suggestive of gas bubbles. A transient loss of the flow-velocity signal occurred (B) during hypothermic circulatory arrest. Establishment of retrograde cerebral perfusion through the superior vena cava was documented by a low-velocity signal of opposite direction (ie, below the baseline). C, This retrograde flow is shown. The signal has been inverted to facilitate automatic velocity measurement and trending. After removal of cerebral air, effective pulsatile antegrade flow was restored (D).
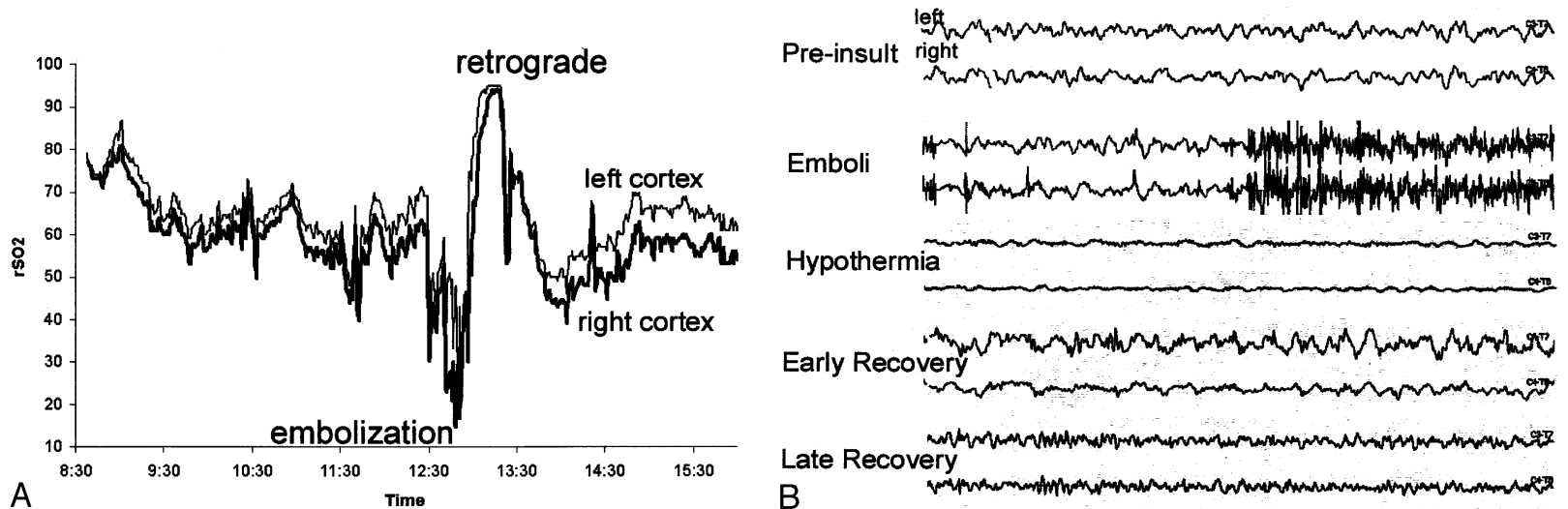

Figure 2. A, Changes in regional cerebral oxygen saturation (rSO2) associated with air embolization and its correction. The thick line denotes the value from the right frontal cortex, and the thin line represents the value from the left frontal cortex. Note the transient decline in both hemispheres accompanying embolization. Subsequent hyperoxia occurred with flow restoration in a cold-suppressed brain. The later new asymmetry suggested oxygen imbalance in the right frontal cortex. B, Responses of cortical synaptic function to embolization, cooling, and gradual improvement in cortical perfusion and oxygenation. Waveform pairs were obtained from left and right frontotemporal derivations, respectively. The initially symmetric preinsult pattern was disrupted with highfrequency artifact, coincident with the appearance of ultrasonographic evidence of gaseous emboli in the flow-velocity spectrum shown in Figure 1, A. Rapid cooling to $26^{\circ} \mathrm{C}$ for neuroprotection suppressed synaptic activity and produced a near-flatline EEG. Oxygen imbalance in the right hemisphere $(A)$ was associated with a new EEG asymmetry as well. Both the amplitude and median frequency of the right hemispheric waveform are decreased. The following day, restoration of symmetric high-frequency EEG activity has occurred. 
might not be universally recognized. ${ }^{4,5}$ Transcranial Doppler monitoring documented both retrograde flow through the middle cerebral artery and later restoration of antegrade flow. Cerebral oximetry identified restoration of adequate oxygenation in the left, but not the right, hemisphere. This physiologic imbalance was additionally noted by the developing EEG asymmetry.

Because class I evidence is lacking, the actual probability of brain injury associated with untreated, new, intraoperative major neurophysiologic abnormalities is unknown. However, our recent retrospective analysis ${ }^{3}$ noted 10 major untreated EEG and coexisting cerebral oxygen abnormalities in a sample of 759 adult and pediatric cardiac procedures. Nine $(90 \%)$ of these 10 patients experienced serious brain injury. Only one injury occurred without a neuromonitoring abnormality. Thus despite some imperfection, the predictive capacity of neuromonitoring during cardiac surgery appears to be very good.

In summary, this case illustrates the key role played by multimodality neuromonitoring in injury prevention. Information provided by the monitoring array promptly detected a physiologic imbalance, determined its probable cause, and objectively assessed immediate patient response to corrective action. The case details suggest that as a result of this specialized monitoring, a serious neurologic complication might have been averted.
The EEG machine used in this clinical summary was provided by a grant from the WHAS Crusade for Children. Neuromonitoring services are provided to all patients undergoing cardiac surgery at no cost by Kosair Children's Hospital. Somanetics Corporation provided the cerebral oximeter and sensors for this case, as well as sponsoring a portion of the group's research.

\section{References}

1. Shanewise JS, Hug CC Jr. Anesthesia for adult cardiac surgery. In: Miller RD, editor. 5th ed. Philadelphia: Churchill-Livingstone; 2000. p. 1753-804.

2. Gundry SR, Razzouk AJ, del Rio MJ, Shirali G, Bailey LL. The optimal Fontan connection: a growing extracardiac lateral tunnel with pedicled pericardium. J Thorac Cardiovasc Surg. 1997;114:552-8.

3. Sehic A, Austin EH III, Yeh T, Pollock SB Jr, Edmonds HL Jr. Fosphenytoin-methylprednisolone neuroprotection during cardiac surgery [abstract]. Proc Eur Neurol Soc. 2002;12:378.

4. Mills NL, Ochsner JL. Massive air embolism during cardiopulmonary bypass. J Thorac Cardiovasc Surg. 1980;80:708-17.

5. Brown JW, Dierdorf SF, Moorthy SS, et al. Venoarterial cerebral perfusion for treatment of massive arterial air embolism. Anesth Analg. 1987;66:673-4. 